Article

\title{
Conceptual Framework of an Intelligent Decision Support System for Smart City Disaster Management
}

\author{
Daekyo Jung ${ }^{1}$, Vu Tran Tuan ${ }^{1}$, Dai Quoc Tran 2 ${ }^{\circledR}$, Minsoo Park ${ }^{2}$ and Seunghee Park ${ }^{2, *}$ \\ 1 Department of Convergence Engineering for future City, Sungkyunkwan University, Suwon 16419, Korea; \\ jdaekyo@skku.edu (D.J.); vu56cd2@gmail.com (V.T.T.) \\ 2 School of Civil, Architectural Engineering \& Landscape Architecture, Sungkyunkwan University, \\ Suwon 16419, Korea; daitran@skku.edu (D.Q.T.); pmskku@naver.com (M.P.) \\ * Correspondence: shparkpc@skku.edu
}

Received: 28 November 2019; Accepted: 13 January 2020; Published: 17 January 2020

\begin{abstract}
In order to protect human lives and infrastructure, as well as to minimize the risk of damage, it is important to predict and respond to natural disasters in advance. However, currently, the standardized disaster response system in South Korea still needs further advancement, and the response phase systems need to be improved to ensure that they are properly equipped to cope with natural disasters. Existing studies on intelligent disaster management systems (IDSSs) in South Korea have focused only on storms, floods, and earthquakes, and they have not used past data. This research proposes a new conceptual framework of an IDSS for disaster management, with particular attention paid to wildfires and cold/heat waves. The IDSS uses big data collected from open application programming interface (API) and artificial intelligence (AI) algorithms to help decision-makers make faster and more accurate decisions. In addition, a simple example of the use of a convolutional neural network (CNN) to detect fire in surveillance video has been developed, which can be used for automatic fire detection and provide an appropriate response. The system will also consider connecting to open source intelligence (OSINT) to identify vulnerabilities, mitigate risks, and develop more robust security policies than those currently in place to prevent cyber-attacks.
\end{abstract}

Keywords: decision support system; big data; artificial intelligence; Internet of Things; disaster management

\section{Introduction}

Urban climate change is an important research issue that must be investigated using multidisciplinary approaches that include both engineering and socio-environmental sciences [1]. An effective approach to strengthen disaster reduction strategies is to build a decision support system (DSS) for each region. A DSS is based on the correlation between the infrastructure, industries, and related communities in a region. Information on the technical, social, and economic properties of a region is collected to establish an effective natural disaster prevention strategy. By analyzing the collected data, we can easily identify the vulnerabilities. Disaster-preparedness strategies for all citizens will make it easier to prepare and respond to real disasters.

\section{Decision Support System}

A DSS is an information system that supports decision-making by selecting the best option by developing and comparing multiple alternatives to solve various issues. DSSs have a long history of development. From the late 1950s to the early 1960s at the Carnegie Institute of Technology, Nutt studied the theory of decision-making at an organization [2]. In the 1960s, MIT conducted technical research related to reciprocating computer systems. Subsequent studies by Pomerol and Adam 
contributed to the development of the DSS concept [3]. In 1981, Bonczek, Holsapple, and Whinston established a DSS framework in a book entitled "Foundations of Decision Support Systems" [4]. Similarly, in 1988, Turban et al. [5] proposed a system to solve semistructured and unstructured problems. This type of effective decision-making by combining the information gained from data processing, learning, and decision-making experience is known as "intelligence DSS." Today, DSSs are proven to be an effective support system in businesses. In addition, DSSs have been the foundation for the development of numerous hypotheses and tools, such as artificial intelligence (AI), human-computer interaction, simulation methods, software engineering, and telecommunication for applications in DSSs. The use of DSSs has grown rapidly since the 1950s, particularly with the aid of effective data analysis tools, enabling improved decision-making using data and information. Several attempts are being made by scientists to improve the effectiveness of decision-making by combining technologies from related fields.

The components of a DSS include data management, model management, user interface, knowledge management, and users, as presented in Figure 1. A DSS is an interactive system that allows decision-makers to easily analyze and evaluate decision models and to process the data to solve complex, unstructured, and nonrepetitive decision-making tasks. The decision-makers access the system via the interface and management components and extract various types of data and information from the database model according to their requirements.

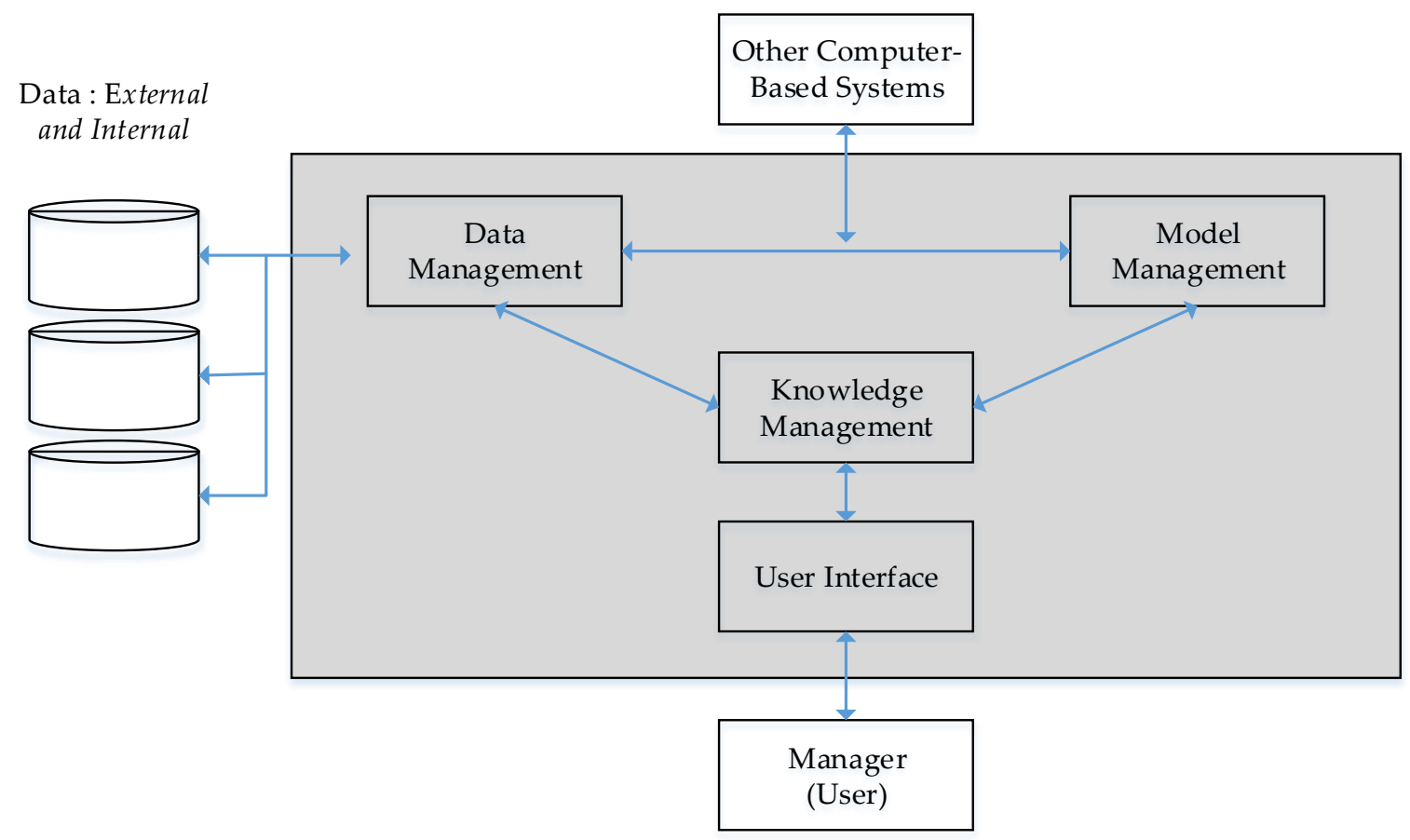

Figure 1. Decision support system components.

(a) Data Management: The data management component comprises a database that stores various data for decision-making as well as a database management system (DBMS). The function of the DBMS in a DSS is to store and provide the data for the decision-making.

(b) Model Management: The model management component comprises a model base that stores various models necessary for the decision-making as well as a model base management system (MBMS). Particularly, the MBMS plays a key role in decision support by providing functions to develop, modify, and control the models required for the decision-making.

(c) User Interface: The user interface is the module system providing an interface between the user and the system for importing and exporting data and performing various analytical procedures. It is also known as the dialogue generation and management software (DGMS) because it provides 
user-friendly and dialogue functions that are easy to understand and use, via menus or graphics processing formats.

(d) Knowledge Management: This module provides quantitative information about the relationships between complex data. Knowledge management provides decision-makers with knowledge and alternative solutions for problem solving. It also signals to the decision-makers if there is a difference between the predicted result and the actual result.

(e) Users: The users who use a DSS are primarily the managers who are responsible for important business decisions. They choose the most appropriate model from the model base, enter the necessary data from the database or import them directly into the model, and then evaluate and analyze the options to determine the best alternative.

This paper is organized as follows. Section 2 presents a literature review about the applications of DSSs, AI, and big data for disaster management. Section 3 discusses the methodology regarding the conceptual framework of an intelligent DSS (IDSS). It also introduces the recent problems of heatwaves, cold waves, and wildfires, and then proposes an AI IDSS system. Finally, Section 4 presents the results and future research.

\section{Literature Review}

\subsection{DSS for Disaster Management}

Although the use of DSSs to support disaster recovery efforts has provided tremendous benefits, these systems have certain disadvantages. One of the disadvantages is the inability to freely and rapidly transfer data between individuals and organizations. This is because the systems and technologies of different organizations are different or incompatible. Nonetheless, DSSs have overcome these disadvantages and are widely used by managers. Rajabifard et al. [6] used an intelligent disaster DSS (IDDSS) as a platform to integrate road, traffic, geographic, economic, and meteorological data. IDDSSs are used in the management of road networks during floods. To prevent hazardous traffic scenarios, they provide the law enforcement with the exact locations to establish traffic management points (TMPs) during an emergency. In 2011, Ishak et al. [7] created a conceptual model of a smart DSS for reservoir operations in case of emergencies such as heavy rainfall. This model can help reservoir operators make accurate decisions for releasing reservoir water so that there is sufficient space for the released water, to avoid local flooding. Moreover, AI has also been integrated into DSSs to increase the decision-making efficiency. Dijkstra's algorithm can find the shortest path between two points and has several applications in various areas. This algorithm has been widely employed in forest fire simulations [8] and in improving the efficiency of route planning [9]. In 2011, Akay et al. [10] improved this algorithm using the Geographic Information System (GIS) to assist firefighters in determining the fastest and safest access routes. This system requires numerous spatial databases, including those of road systems and land. Barrier systems have also been established to simulate the scenario of banned roads; hence, these systems are not only used to determine the fastest route but also to assist firefighters in identifying unforeseen scenarios and determining safe and reliable routes.

To deal with disasters, we require an effective disaster management system. However, disaster management systems depend on various data and information and knowledge of previous disasters. Therefore, coordination between the relevant agencies is necessary to integrate the data effectively. A DSS is unable to prevent all catastrophic damage; however, it can mitigate the potential risks by developing early warning strategies and preparing appropriate options. Particularly in the context of disaster management [11], the process of developing intelligent systems focuses on the methods of transferring expertise from human experts to computers and implementing theoretical models based on the transferred knowledge. The model proposed by Turban (Figure 2) included the five main activities of DSS components [12]. Within the scope of AI, there are several methods for solving interdependent problems. Examples of alternative methods include Bayesian networks, which approximate reasoning (fuzzy logic), and metadata (artificial neural networks, machine learning, genetic algorithms, and 
swarm intelligence), which involves the use of a hybrid approach combining the fuzzy IF-THEN rules and optimization techniques to collect and present inaccurate information [13].

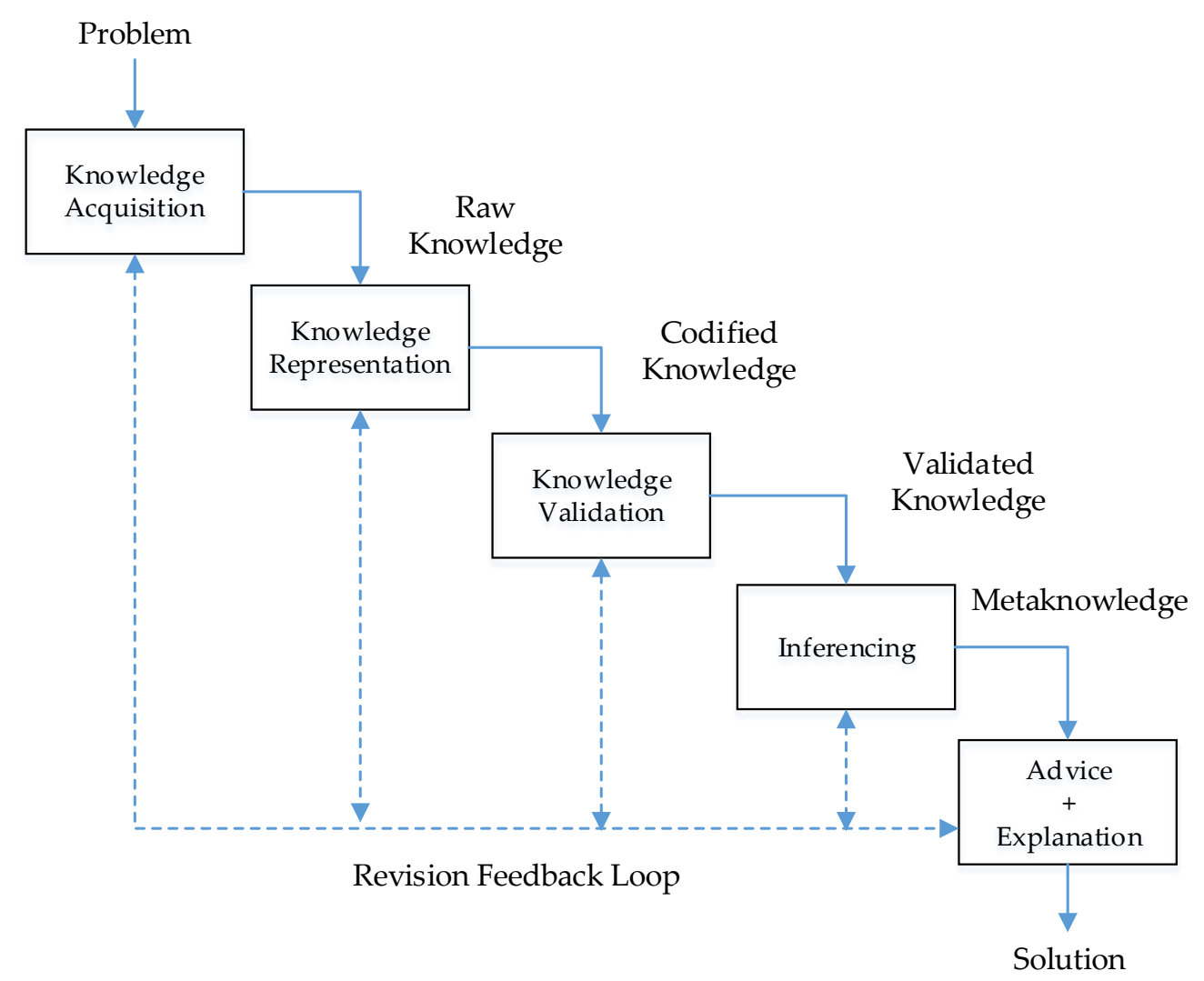

Figure 2. The process of knowledge engineering (adapted).

\subsection{AI for Disaster Management}

$\mathrm{AI}$ is used in various applications, such as customer service, transactions, and healthcare. Recently, researchers have found that AI can be employed to predict disasters. With numerous high-quality datasets, AI can predict the occurrence of natural disasters, which can assist in saving the lives of thousands of people and in preventing financial losses. Natural disasters that can be predicted by AI include earthquakes, floods, storms, forest fires, and volcanic eruptions. In the field of earthquake prediction, researchers are collecting vast amounts of seismic data for analysis using deep-learning systems. Researchers use the data collected from past earthquakes to make improved predictions for future earthquakes. Alarifi et al. [14] proposed an AI prediction system based on artificial neural networks, which could be used to predict the magnitude of an earthquake. AI can also employ seismic data to analyze the magnitude and pattern of an earthquake, as well as to aid in predicting the occurrence of an earthquake. Goymann et al. [15] presented data for the establishment, training, and evaluation of a neural network to detect flood and water levels. AI-based systems can learn rainfall records, climate records, and flood simulation tests and can predict floods better than traditional systems.

\subsection{Big Data for Disaster Management}

Big data result from the large amount of data generated by the explosive growth of information technology and communications, as well as by the rapid spread of smart devices $[16,17]$. They have been applied in several fields $[18,19]$. Using large datasets, it is possible to analyze past disaster data and develop future scenarios for the possibilities of disasters and the damage caused by them [20]. Big data contribute to the science of decision-making by developing a technology that can read common patterns of user behaviors and predict future steps [21]. AI is defined as a computer program that can 
think and process information like a human being. It is the science that enables computers to perform intellectual activities based on experience and knowledge. Existing data serve as the criteria for core judgments, and an AI learning algorithm narrows the choices for decision-making and provides feedback that is very similar to human judgment. For an optimal response to complex disasters, a large amount of data should be analyzed and used; hence, AI can be used to understand large-scale social phenomena [22]. By big data analysis and simulation, the selection of the required actual data can be achieved using the judgment of AI based on the existing empirical data.

\subsection{Intelligent Decision Support Systems}

Using AI, researchers have attempted to improve the effectiveness of decision-making. Integrated AI support systems or IDSSs have been created and used in various fields, such as healthcare and commerce.

These systems use AI tools to infer, learn, memorize, plan, and analyze the data. Simon presented four stages in a decision-making process, which are displayed in Figure 3 [23]. Intelligence is the first stage of the study and requires the decision-makers to develop an understanding of the problem and collect information related to the decision-making. Similarly, the design stage involves characterizing the data with important variables based on decision issues, determining the criteria for the decisions, and developing decision models that can be used to evaluate alternative decisions. In the third stage, the decision-makers review the alternatives and choose the consolidated decisions that satisfy the alternatives. The final stage, sometimes referred to as evaluation, is when the decision-makers assess the consequences of a decision.

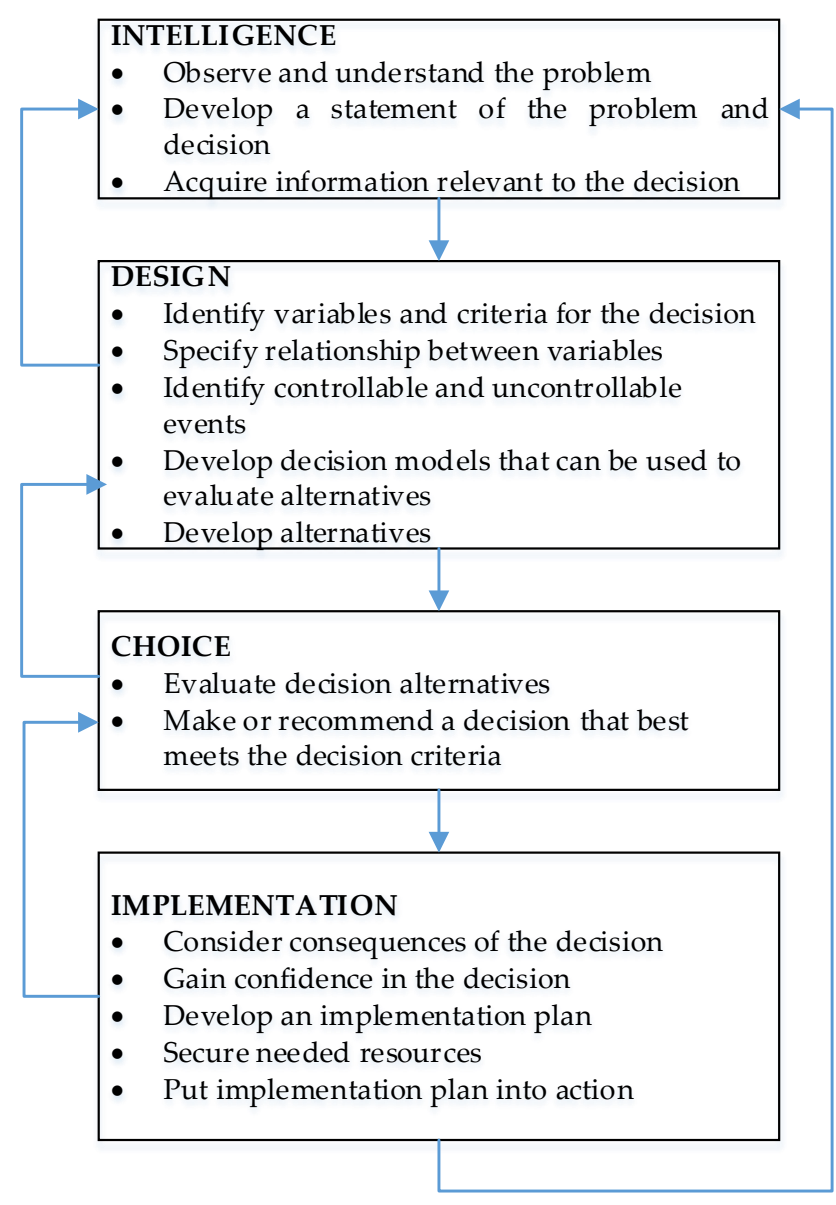

Figure 3. Four decision-making stages 
IDSSs utilize AI techniques to provide better support to decision-makers. AI tools, such as fuzzy logic, case-based reasoning, evolutionary computing, artificial neural networks, and intelligent agents, when combined with a DSS provide powerful methods for solving difficult problems that are frequently real-time and involve large amounts of distributed data [24]. IDSSs utilize some of the below-mentioned tools:

Fuzzy logic for intelligent decision support;

Expert systems for intelligent decision support;

Evolutionary computing for intelligent decision support;

Intelligent agents for intelligent decision support.

Disaster response is a continuous decision-making process based on past experiences, because a disaster scenario is recognized as a disaster until it ends. In the past, because of the limitations regarding information and data-collection technology, the decision-making process had various constraints and relied on the subjective experiences of the decision-makers. Recently, however, information technology has developed considerably and the amount of information that can be used to make decisions has increased sharply. The information processing ability of humans is limited, and responding to disasters requires timely decisions. The benefits of combining a DSS and AI to create an IDSS in disaster management are clear. However, the use of information of past disasters to analyze and make predictions has not sufficiently been investigated. Therefore, this study aims to contribute to this growing area of research by exploring a conceptual framework that supports intelligent decision-making using big data to help decision-makers make more rapid and more accurate decisions.

Recently, a series of innovative solutions based on free and open-source software has been developed [25-27]. Open source information is data collected from publicly available sources and the system data. In this study, the proposed system also collected data from various open sources. With the growth of big data and online social networks, increasing amounts of data are available online, which creates opportunities for criminals-particularly adversaries preparing for a cyberattack [28]. Thus, an IDSS can become vulnerable to cyberattacks. Consequently, the scientific community, companies, practitioners, and governments worldwide are searching for solutions to mitigate risks [29]. Hayes and Cappa [30] introduced open source intelligence (OSINT) to provide tactical and strategic recommendations for organizations to prevent cyberattacks by identifying vulnerabilities, mitigating risks, and formulating robust security policies.

\section{Methodology}

Heat waves, cold waves, and wildfires are the main concerns for the South Korean government, because of the difficulty in predicting and responding to these events. Therefore, the proposed AI IDSS focuses on the aforementioned phenomena. In the next two subsections, the issues and scenarios regarding heat waves, cold waves, and wildfire phenomena in South Korea are briefly introduced. Based on these issues, an AI IDSS with a multifunction AI algorithm is presented.

\subsection{Issues and Scenarios of Heatwave/Cold Wave Disasters}

The fund for disaster support money paid to the families of those who are killed by heat is limited between 5000 and 10,000 dollars (in the case of household heads) based on the South Korea disaster relief regulations and costs (DRRCs) for disaster recovery. However, no system or technology has been established to objectively determine the causes of deaths during the summer in South Korea. In addition, there have been numerous difficulties in diagnosing the causes of deaths owing to victim interventions and ethical issues that may have arisen. Furthermore, while heatwave damage occurs in almost all areas during the summer, the extent of the damage varies depending on the local scenarios and personal health conditions, which can lead to legal repercussions. 
A domestic cold wave alarm is issued based on three criteria. It is issued during the winter (October to April), when the minimum temperature the following morning is expected to drop by more than $10^{\circ} \mathrm{C}$ compared to that of the previous day and is below $3{ }^{\circ} \mathrm{C}$, or when the temperature is lower than the average winter temperature values of previous years by $3^{\circ} \mathrm{C}$. The warning is also issued when the minimum morning temperature is expected to be below $12{ }^{\circ} \mathrm{C}$ for two days or more, or when there is a significant loss of life and property due to a sudden fall in the temperature. If there is a specific list of victims or temperature damages, it is possible to set an absolute standard for facility damages or loss of life. However, as such a database has not yet been established, cold weather management will need to be implemented and systematically managed by generating the associated database.

There is no big data AI study case for heat and cold waves. In 2018, the Ministry of Public Administration and Security identified heat and cold waves as new types of disasters. Currently, there is no specific management standard to deal with crises caused by heat and cold waves in Korea. It is also necessary to improve the speed and accuracy of the system that predicts heat and cold waves. For example, it is possible to predict pre-heat phenomena two days in advance; however, in developed regions such as Europe or the U.S., this can be done three to five days in advance. Through this study, by collecting data to prevent and be prepared for disasters, an AI-based support system was developed to enable more rapid and more accurate decision-making than is currently achievable.

\subsection{Issues and Situations Of Forest Fire Disaster}

When considering information on the direction and speed of a spreading fire, a possibility of widespread fire is found to exist. Accordingly, a rapid plan to protect nearby houses and evacuate people is required. However, a gap remains in relaying the wildfire information from the fire helicopter to the ground firefighter commander. Even if a firefighter commander decides that an emergency defense is needed, it is still necessary to go through the integrated fire protection procedure to adopt the necessary measures. In the process, damages are frequently increased because of the inappropriate propagation of information or lack of content transformation. Therefore, it is necessary to improve the information sharing system for handling forest fires.

\subsection{A System Using Big Data and AI for Intelligent Decision Support}

The level of success of a DSS is assessed using two parameters. The first parameter is the data input rate and output rate, which represent the information that is useful for the decision-making process. The second parameter is time. To improve the efficiency of the DSS in this study, a conceptual framework using big data and AI to enhance the above-mentioned parameters is proposed. The decision points of the standard manual for heat/cold wave and forest fire crisis management and the services provided by the decision-maker in the event of a disaster are summarized in Figure 4, along with the big data system processes and big data analysis. The proposed big data process is composed of data sources, collection, storage, analysis, and presentation order, and is built on the basis of the data lake (DL) form. The data collection is performed based on the type of natural disaster that a given DSS is built to deal with. The choice of data source for the analysis is extremely important. If the collected data are not reliable, they will be difficult to process and discard. Extreme disasters, such as heat and cold waves, need to be analyzed, forecasted, and planned for in advance. By contrast, with forest fires, the post-disaster response includes population evacuation, which is more important than forecasting. Therefore, camera data should be analyzed to predict the speed and direction of the flames and to send information to the people in the affected areas. 


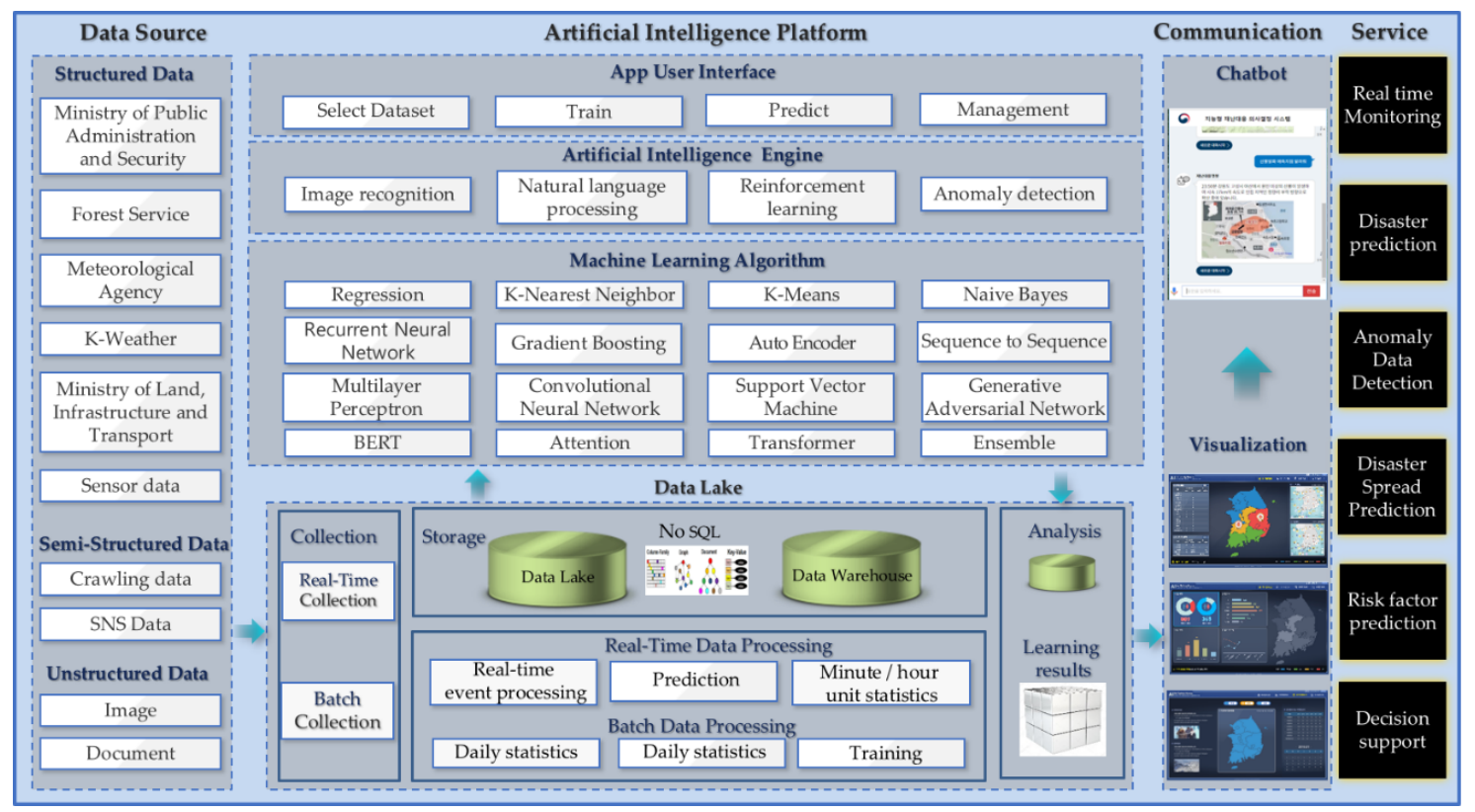

Figure 4. System using big data and AI for intelligent decision support.

To collect and store data, it is important to build a DL. A DL differs from a data warehouse (DW) in that the former contains both structured and unstructured data. Post data collection, it undergoes the process of extraction, transformation, and loading (ETL); however, this process is time-consuming and makes it difficult to arrive at a timely decision. Hence, this system splits the DL archive into two parts. The first is a platform that can store the data in the original form without going through the ETL process, and the second is the data analysis platform that undertakes the ETL process. The collected data include real-time data and batch data. Real-time data are structured data, which can be periodically collected and processed by the existing DWs. Batch data are the raw and unstructured data that need to be used for analysis.

\subsection{Data Collection and Storage}

Essentially, most structured data are collected by an open API. The data are provided in Comma-Separated Values (CSV) format on a public data portal operated by the Ministry of Administration and Security. Because big data systems use data from various organizations, the range of the collected data is wide. However, only the data considered necessary for the analysis must be used. In the case of image data that require real-time processing, satellite image information can be used to cover a wide area. Along with satellite images, Closed-Circuit Television (CCTV) cameras can collect image data anytime and anywhere, and they can be used to detect a fire early. Most CCTV cameras in South Korea are used for traffic or crime prevention, and their data storage cycle is only 30 days. Thus, their application to machine learning in the disaster field is limited. These elements are listed in Table 1, which describes the types of disaster, data sources, and organizations responsible for answering the questions of decision-makers and enabling them to make rapid and precise decisions. 
Table 1. Questions and data sources required for disaster response (Application Programming Interface (API), Closed-Circuit Television (CCTV), Unmanned Aerial Vehicle (UAV)).

\begin{tabular}{|c|c|c|}
\hline Disaster Type & Data Source & Question \\
\hline Extreme Weather & $\begin{array}{c}\text { Statistical Geographic Information } \\
\text { Service (Open API) }\end{array}$ & $\begin{array}{l}\text { Current status of population and households } \\
\text { in heat/cold wave expected areas }\end{array}$ \\
\hline Extreme Weather & $\begin{array}{l}\text { Statistical Geographic Information } \\
\text { Service (Open API) }\end{array}$ & $\begin{array}{l}\text { Status of land use and age of buildings in } \\
\text { heat/cold wave expected areas }\end{array}$ \\
\hline Extreme Weather & Data Portal (Open API) & $\begin{array}{l}\text { Status of operation of hot/cold shelter in } \\
\text { heat/cold wave expected areas }\end{array}$ \\
\hline Extreme Weather & $\begin{array}{l}\text { Korea Centers for Disaster Control } \\
\text { and Prevention (Document) }\end{array}$ & $\begin{array}{c}\text { Cases of human damage from past } \\
\text { heat/cold waves }\end{array}$ \\
\hline $\begin{array}{l}\text { Extreme Weather } \\
\text { Wildfire }\end{array}$ & $\begin{array}{l}\text { Korea Meteorological Administration } \\
\text { (Open API) }\end{array}$ & $\begin{array}{l}\text { Weather status (temperature, precipitation, } \\
\text { wind, humidity, atmospheric pressure) }\end{array}$ \\
\hline $\begin{array}{l}\text { Extreme Weather } \\
\text { Wildfire }\end{array}$ & CCTV, UAV, Satellite (Image, Video) & Disaster image information \\
\hline Heat Wave & K-Water (Open API) & $\begin{array}{l}\text { Status of water supply in heat/cold wave } \\
\text { expected areas }\end{array}$ \\
\hline Wildfire & Data Portal (Open API) & Forest fire danger rating index \\
\hline Wildfire & Data Portal (Open API) & Past wildfire disaster cases \\
\hline Wildfire & $\begin{array}{c}\text { Korea Database on Protected Areas } \\
\text { (CSV) }\end{array}$ & $\begin{array}{l}\text { Status of cultural assets and hazardous } \\
\text { facilities near the forest area }\end{array}$ \\
\hline
\end{tabular}

\subsection{Machine Learning Based on a Data Analysis Algorithm for AI Management}

The algorithm modeled for the machine learning data analysis is displayed in Figure 5. It uses convolutional neural network (CNN) and generative adversarial network (GAN) algorithms for natural disaster detection and employs reinforcement learning (RL) to predict wildfire direction. A CNN is a specific type of artificial neural network that uses perceptron, a machine learning unit algorithm, to analyze the data. A CNN can be applied to image processing, natural language processing, and other types of cognitive tasks.

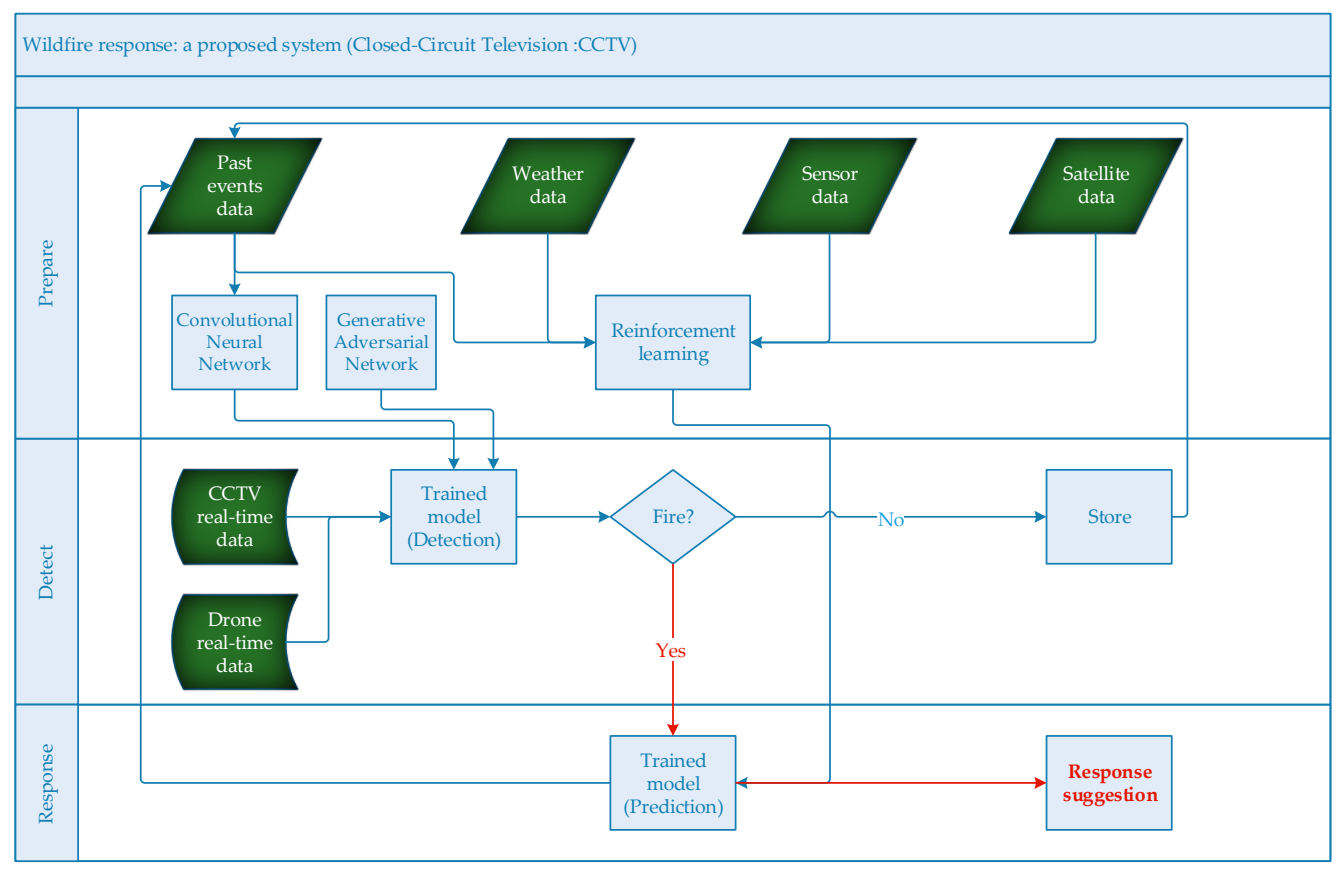

Figure 5. Wildfire response: the proposed system diagram. 
The GAN algorithm, which is an unsupervised learning algorithm used in the model, utilizes image data both from fires and from normal conditions for learning. Real-time CCTV and Unmanned Aerial Vehicle (UAV) data are then fed into and trained in the models to determine the occurrence of a fire. If it is not a forest fire, then the used data will be stored and utilized for subsequent training. In the case of forest fires, significant information about the weather and real-time satellite images are required. The weather data include the temperature, wind speed, wind direction, rainfall, and humidity. At this point, the model is transformed into an enhanced model that predicts the propagation speed and the direction of the fire per unit of space.

The following discussion presents how the proposed algorithm can be used for fire detection and response. CNNs were first introduced by LeCun et al. [31]. By collective knowledge from the training data, computers can learn and understand a structure based on a hierarchy of concepts. A common $\mathrm{CNN}$ architecture contains various types of processing layers including convolution, pooling, and fully connected layers [32]. The optimization process of a CNN is conducted by trial and error and guided by checking the validation set error [33]. In this study, the overall architecture and layers of FireNet [33] were used. The FireNet CNN architecture contains three convolutional layers of sizes 64,128 , and 256, with kernel filters of sizes $5 \times 5,4 \times 4$, and $1 \times 1$, respectively. Each convolutional layer is followed by a max-pooling layer with a kernel size of $3 \times 3$ and local response normalization. This set of convolutional layers is followed by two fully connected layers, each with 4096 incoming connections and a hyperbolic tangent activation function. A dropout of 0.5 is applied across these two fully connected layers during the training to offset the residual over-fitting. Finally, we obtain a fully connected layer with two incoming connections and a soft-max activation output [33].

The experiment was performed using a dataset of 9852 images from Chenebert et al. [33], and the testing was conducted on 2931 images.

The experiment was performed using an NVIDIA GeForce GTX 2080 Ti with 12 GB onboard memory and a deep learning framework [34] on an Intel Xeon CPU E3-1230 v6 with 32 GB RAM. From the results, it was noted that FireNet could predict the test data with an accuracy of $91.2 \%$. Figure 6 displays the grad class activation map (CAM) results, elucidating how the computer differentiated between a fire and a non-fire. This demonstrates that this algorithm can be used for automatic fire detection and can suggest the appropriate response.
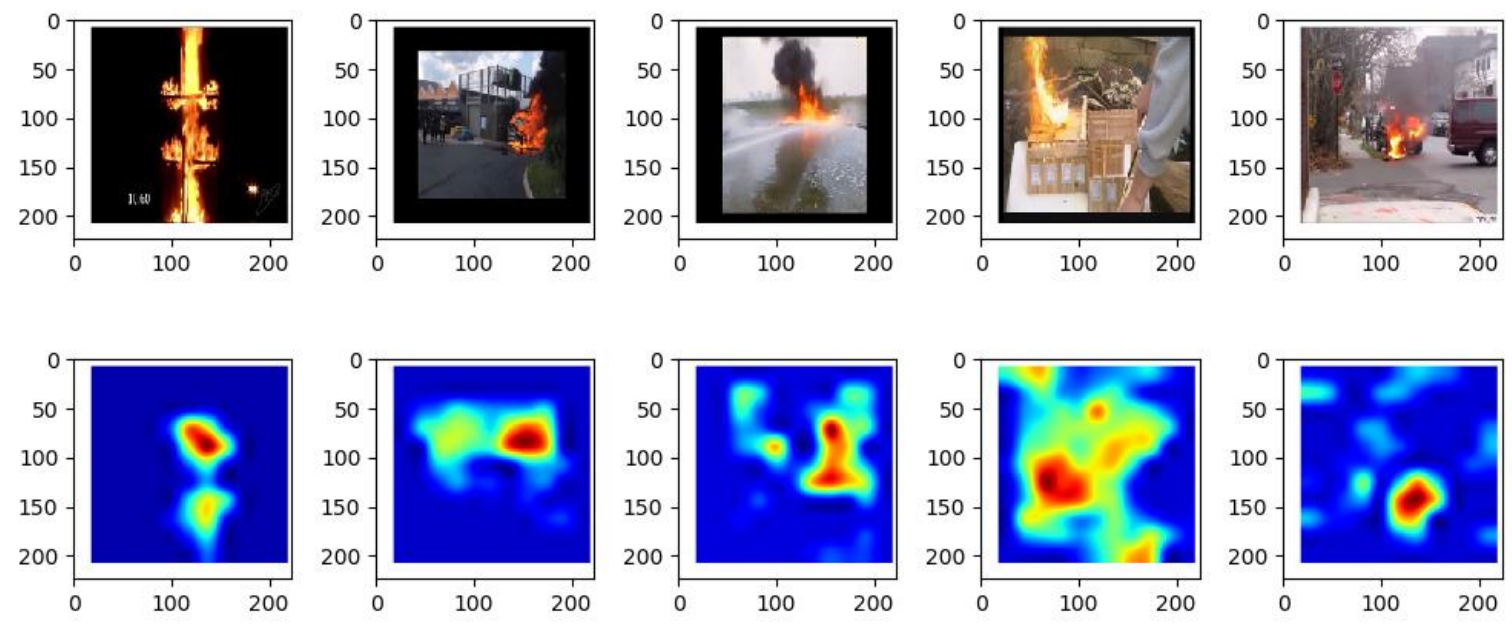

Figure 6. FireNet model localizes the fire area using class activation maps (CAMs), which highlight the areas of the image that are most important for making predictions.

\section{Conclusions}

With climate change, natural disasters have increased in frequency and intensity compared to those in the past. Thus, in this study, we proposed a support system for disaster response decisions using big data storage and machine learning analysis. We focused on forest fires and hot/cold disasters, 
which cause significant loss of life and property annually. Current disaster area systems focusing on simple methods of information collection, efficient visualization, storage systems, and big data analysis are inadequate. In other countries, big data analysis conducted during the entire process of disaster management, including prevention, preparation, response, and recovery, mainly uses video data and social media. Based on previous studies, we developed a big data analysis algorithm to support decisions for heat wave disasters and proposed its application. In the field of disaster management, which requires prompt decisions, if the current forest fire detection system employs image recognition technology intelligently, preventive responses can reduce the damage. In addition, it can provide information that may be useful for decision-making, instead of yielding simple statistical information. Moreover, a simple example of using a CNN to detect fire in a surveillance video was introduced. It was evident that this algorithm could be used for automatic fire detection and provide an appropriate response. This conceptual framework can support decision-makers and enable them to make correct decisions that can save lives, reduce property damage, and assist in predicting disasters. In the future, the system and the algorithm will be tested with real events to gradually increase its accuracy by diversifying the input data and correcting the algorithms. We will also consider connecting the system with OSINT to identify vulnerabilities, mitigate risks, and formulate more robust security policies than the current ones to prevent cyberattacks.

Author Contributions: D.J. Developed Ideas and System Modeling; V.T.T. Methodology and Manuscript Writing; D.Q.T. Methodology and Edited the Paper; M.P. Project administration; S.P. Supervision and Funding Acquisition. All authors have read and agreed to the published version of the manuscript.

Funding: This research was supported by a grant [2019-MOIS31-011] from the Fundamental Technology Development Program for Extreme Disaster Response funded by the Ministry of Interior and Safety [MOIS, Korea] and supported by the Korea Ministry of Land, Infrastructure and Transport [MOLIT] as an [Innovative Talent Education Program for Smart City].

Conflicts of Interest: The authors declared no conflict of interest.

\section{Abbreviations}

The following abbreviations are used in this manuscript:

$\begin{array}{ll}\text { Decision Support System } & \text { DSS } \\ \text { Big Data } & \text { BD } \\ \text { Internet of Things } & \text { IOT } \\ \text { Database Management System } & \text { DBMS } \\ \text { Model Base Management System } & \text { MBMS } \\ \text { Dialogue Management and Management Software } & \text { DGMS } \\ \text { Intelligent Disaster Decision Support System } & \text { IDDSS } \\ \text { Traffic Management Points } & \text { TMP } \\ \text { Artificial Intelligence } & \text { AI } \\ \text { Intelligent Decision Support System } & \text { IDSS } \\ \text { Open Source Intelligence } & \text { OSINT } \\ \text { Disaster Relief Regulations and Costs } & \text { DRRC } \\ \text { Data Base } & \text { DB } \\ \text { Data Lakes } & \text { DL } \\ \text { Extraction, Transformation, and Loading } & \text { ETL } \\ \text { Social Networking Service } & \text { SNS } \\ \text { User Interface } & \text { UI } \\ \text { k-Nearest Neighbor } & \text { KNN } \\ \text { Convolutional Neural Network } & \text { CNN } \\ \text { Recurrent Neural Network } & \text { RNN } \\ \text { Multilayer Perceptron } & \text { MLP } \\ \text { Support Vector Machine } & \text { SVM } \\ \text { Generative Adversarial Network } & \text { GAN } \\ \text { Bidirectional Encoder Representations from Transformers } & \text { BERT }\end{array}$


Data Warehouse $\quad$ DW

Comma-Separated Values $\quad$ CSV

Closed-Circuit Television CCTV

Class Activation Maps CAMs

Unmanned Aerial Vehicle UAV

Application Programming Interface API

\section{References}

1. Pisello, A.L.; Rosso, F.; Castaldo, V.L. The role of building occupants' education in their resilience to climate-change related events. Energy Build. 2017, 154, 217-231. [CrossRef]

2. Nutt, P.C.; Wilson, D.C. Handbook of Decision Making; John and Wiley \& Sons: Hoboken, NJ, USA, 2010.

3. Pomerol, J.-C.; Adam, F. Practical Decision Making-From the Legacy of Herbert Simon to Decision Support Systems. In Actes de la Conference Internationale IFIP TC8/WG8.3; The Monash University Decision Support Systems Laboratory: Prato, Italy, 2004; Volume 3, pp. 647-657.

4. Bonczek, R.H.; Holsapple, C.W.; Whinston, A.B. Foundations of Decision Support Systems; Academic Press: Cambridge, MA, USA, 2014.

5. Turban, E.; Cameron Fisher, J.; Altman, S. Decision support systems in academic administration. J. Educ. Adm. 1988, 26, 97-113. [CrossRef]

6. Rajabifard, A.; Thompson, R.G.; Chen, Y. An intelligent disaster decision support system for increasing the sustainability of transport networks. Nat. Resour. Forum 2015, 39, 83-96. [CrossRef]

7. Ishak, W.H.W.; Ku-Mahamud, K.R.; Morwawi, N.M. Conceptual model of intelligent decision support system based on naturalistic decision theory for reservoir operation during emergency situation. Int. J. Civ. Environ. Eng. 2011, 11, 6-11.

8. Lopes, A.M.G.; Sousa, A.C.M.; Viegas, D.X. Numerical simulation of turbulent flow and fire propagation in complex topography. Numer. Heat Transf. Part A Appl. 1995, 27, 229-253. [CrossRef]

9. Fan, D.; Shi, P. Improvement of Dijkstra's algorithm and its application in route planning. In Proceedings of the 2010 Seventh International Conference on Fuzzy Systems and Knowledge Discovery, Yantai, China, 10-12 August 2010.

10. Akay, A.E.; Wing, M.G.; Sivrikaya, F. A GIS-based decision support system for determining the shortest and safest route to forest fires: A case study in Mediterranean Region of Turkey. Environ. Monit. Assess 2012, 184, 1391-1407. [CrossRef] [PubMed]

11. Correia, A.; Severino, I.; Nunes, I.L. Knowledge management in the development of an intelligent system to support emergency response. In International Conference on Applied Human Factors and Ergonomics; Springer: Berlin/Heidelberg, Germany, 2017.

12. Turban, E.; Sharda, R.; Delen, D. Decision Support and Business Intelligence Systems (Required); Prentice Hall Press: Upper Saddle River, NJ, USA, 2010.

13. Simões-Marques, M.; Figueira, J.R. How Can AI Help Reduce the Burden of Disaster Management Decision-Making? In International Conference on Applied Human Factors and Ergonomics; Springer: Berlin/Heidelberg, Germany, 2018.

14. Alarifi, A.S.N.; Alarifi, N.S.N.; Al-Humidan, S. Earthquakes magnitude predication using artificial neural network in northern Red Sea area. J. King Saud. Univ. Sci. 2012, 24, 301-313. [CrossRef]

15. Goymann, P.; Herrling, D.; Rausch, A. Flood Prediction through Artificial Neural Networks. In Proceedings of the Eleventh International Conference on Adaptive and Self-Adaptive Systems and Applications, At Venice, Italy, 5-9 May 2019.

16. Ardito, L.; Scuotto, V.; Del, G.M. A bibliometric analysis of research on Big Data analytics for business and management. Manag. Decis. 2019, 57, 1993-2009. [CrossRef]

17. Bean, T.H.D. Paul Barth and Randy How Big Data is Different. MIT Sloan Management Review. Available online: https://sloanreview.mit.edu/article/how-big-data-is-different/ (accessed on 12 September 2019).

18. Vecchio, P.D.; Mele, G.; Ndou, V. Creating value from Social Big Data: Implications for Smart Tourism Destinations. Inf. Process. Manag. 2018, 54, 847-860. [CrossRef]

19. Elia, G.; Polimeno, G.; Solazzo, G. A multi-dimension framework for value creation through big data. Ind. Mark. Manag. 2019. [CrossRef] 
20. Yu, M.; Yang, C.; Li, Y. Big Data in Natural Disaster Management: A Review. Geosciences 2018, 8, 165. [CrossRef]

21. Jeble, S.; Patil, Y. Role of Big Data in Decision Making. Oper. Supply Chain Manag. Int. J. 2018, 11, 36. [CrossRef]

22. Arslan, M.; Roxin, A.; Cruz, C. A Review on Applications of Big Data for Disaster Management. In Proceedings of the 13th International Conference on Signal Image Technology \& Internet Based Systems, Jaipur, India, 4-7 December 2017.

23. Simon, H.A. The New Science of Management Decision; Prentice Hall PTR: Upper Saddle River, NJ, USA, 1960.

24. Phillips-Wren, G. Intelligent decision support systems. In Multicriteria Decision Aid and Artificial Intelligence: Links, Theory and Applications; Wiley-Blackwell: Hoboken, NJ, USA, 2013.

25. Afuah, A.; Tucci, C.L. Value Capture and Crowdsourcing. Acad. Manage. Rev. 2013, 38, 457-460. [CrossRef]

26. Bhatt, P.; Ahmad, A.J.; Roomi, M.A. Social innovation with open source software: User engagement and development challenges in India. Technovation 2016, 52-53, 28-39. [CrossRef]

27. Cappa, F.; Rosso, F.; Hayes, D. Monetary and Social Rewards for Crowdsourcing. Sustainability 2019, 11, 2834. [CrossRef]

28. Lakhani, K.; Wolf, R.G. Why Hackers Do What They Do: Understanding Motivation and Effort in Free/Open Source Software Projects. SSRN Electron. J. 2003. [CrossRef]

29. Cazorla, L.; Alcaraz, C.; Lopez, J. A three-stage analysis of IDS for critical infrastructures. Comput. Secur. 2015, 55, 235-250. [CrossRef]

30. Hayes, D.R.; Cappa, F. Open-source intelligence for risk assessment. Bus. Horiz. 2018, 61, 689-697. [CrossRef]

31. LeCun, Y.; Bottou, L.; Bengio, Y. Gradient-based learning applied to document recognition. Proc. IEEE 1998, 86, 2278-2324. [CrossRef]

32. Goodfellow, I.; Bengio, Y.; Courville, A. Deep Learning; The MIT Press: Cambridge, MA, USA, 2016.

33. LeCun, Y.; Bengio, Y.; Hinton, G. Deep learning. Nature 2015, 521, 436-444. [CrossRef] [PubMed]

34. Chollet, F. Keras: The Python Deep Learning Library; Astrophysics Source Code Library, 2018. Available online: https://keras.io/\#support (accessed on 18 November 2019).

(C) 2020 by the authors. Licensee MDPI, Basel, Switzerland. This article is an open access article distributed under the terms and conditions of the Creative Commons Attribution (CC BY) license (http://creativecommons.org/licenses/by/4.0/). 